\title{
Long-term Safety and Dosing of OnabotulinumtoxinA: A Prospective, Observational Study
}

\author{
Theodore Wein, Mandar Jog, Meetu Bhogal, Sonja Dhani, Robert Miller, \\ Farooq Ismail, Richard Beauchamp, Grace Trentin
}

\begin{abstract}
Background: Although therapeutic treatments are intended to help alleviate symptoms associated with disease, safety must be carefully considered and monitored to confirm continued positive benefit/risk balance. The objective of MOBILITY was to study the long-term safety of onabotulinumtoxinA for treatment of various therapeutic indications. Methods: A prospective, multicenter, observational, Phase IV Canadian study in patients treated with onabotulinumtoxinA for a therapeutic indication. Dosing was determined by the participating physician. Adverse events (AEs) were recorded throughout the study. Results: Patients $(n=1372)$ with adult focal spasticity, blepharospasm, cerebral palsy, cervical dystonia, hemifacial spasm, hyperhidrosis, or "other" diagnoses were enrolled into the safety cohort. Eighty-three patients (6\%) reported 209 AEs; 44 AEs in 24 patients (2\%) were considered treatment-related AEs. Seventy-two serious AEs were reported by 38 patients (3\%); 10 serious AEs in 5 patients $(0.4 \%)$ were considered treatment related. Most commonly reported treatment-related AEs were muscular weakness $(n=7 / 44)$ and dysphagia $(n=6 / 44)$. Conclusions: In patients with follow-up for up to six treatments with onabotulinumtoxinA, treatment-related AEs were reported in $<2 \%$ of the safety population over the course of nearly 5 years. Our findings from MOBILITY provide further evidence that onabotulinumtoxinA treatment is safe for long-term use across a variety of therapeutic indications.
\end{abstract}

RÉSUMÉ: Dosage et sécurité à long terme de l'onabotulinumtoxinA : une étude prospective et observationnelle. Contexte : Bien que les traitements thérapeutiques soient destinés à soulager les symptômes associés à une maladie, il importe d'examiner avec grand soin leur sécurité et d'en assurer un suivi afin de maintenir un rapport bénéfice/risque qui soit positif. L'objectif de MOBILITY a donc été d'étudier la sécurité à long terme de l'onabotulinumtoxinA dans le traitement de plusieurs indications thérapeutiques. Méthodes : Nous avons ainsi fait appel à une étude canadienne prospective et observationnelle menée dans plusieurs centres de santé. Dans cette étude de phase IV, des patients ont été traités avec l'onabotulinumtoxinA en vertu d'indications thérapeutiques. Ce sont des médecins participants qui en avaient déterminé le dosage. De plus, tout événement indésirable a été noté en cours d'étude. Résultats : Au total 1372 patients ont été inclus dans cette cohorte $(\mathrm{n}=1372)$. Ces patients étaient atteints des troubles suivants : spasticité focale chez l'adulte, blépharospasme, infirmité motrice cérébrale, dystonie cervicale, spasmes hémifaciaux, hyperhidrose, etc. On a signalé chez 83 patients, soit $6 \%$ d'entre eux, des événements indésirables. On a aussi estimé que 44 événements indésirables ayant affecté 24 patients ( $2 \%$ ) étaient reliés au traitement proprement dit. Ajoutons que 38 patients ( $3 \%)$ ont signalé avoir été victimes d'événements indésirables et que 10 événements indésirables ont été reliés au traitement chez 5 patients $(0,4 \%)$. Enfin, les événements indésirables les plus communément signalés ont été la faiblesse musculaire $(n=7 / 44)$ et la dysphagie $(n=6 / 44)$. Conclusions : Dans le cas de patients ayant bénéficié de six traitements ou moins avec l'onabotulinumtoxinA, des événements indésirables ont été signalés chez $<2 \%$ d'entre eux au cours des presque cinq prochaines années. Tirés de MOBILITY, nos résultats apportent une preuve additionnelle que les traitements avec l'onabotulinumtoxinA sont à long terme sécuritaires dans le cas de nombreuses indications thérapeutiques.

Keywords: Botulinum toxin, Cerebral palsy, Movement disorders, Spasticity, Dosing, Safety doi: $10.1017 / \operatorname{cjn} .2019 .238$

Can J Neurol Sci. 2019; 46: 742-752

\section{BACKGROUND}

OnabotulinumtoxinA $\left(\right.$ BOTOX $\left.^{\circledR}\right)$ is a purified neurotoxin that acts by blocking presynaptic acetylcholine release, leading to localized reduction of muscle contractions. ${ }^{1}$ This unique mechanism of action led to the investigation of therapeutic uses with the toxin, starting with treatment for strabismus. ${ }^{2,3}$ Since then, the broad applicability of onabotulinumtoxinA has been widely recognized and studied and has been used for over 25 years for many

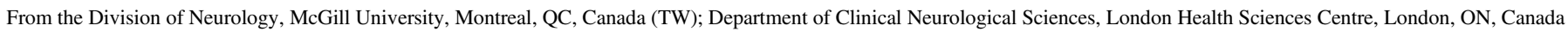

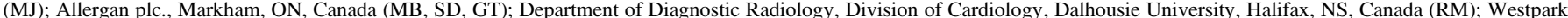

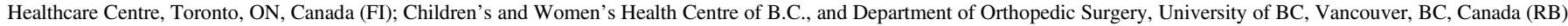
Received January 12, 2018. Final Revisions Submitted June 14, 2019. Date of Acceptance June 17, 2019.

Present address: Sonja Dhani, Purdue Pharma (Canada), Pickering, ON, Canada; Grace Trentin, Akcea Therapeutics Canada, Ottawa, ON, Canada

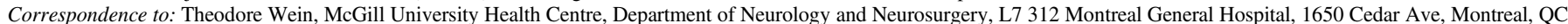
H3G 1A4 Canada. Email: twein@videotron.ca 
therapeutic indications. ${ }^{3}$ More recently, data has illustrated the ability of onabotulinumtoxinA to act not only on efferent pathways but also on afferent pathways, leading to a decrease in pain responses. ${ }^{4,5}$ In Canada, onabotulinumtoxinA is currently approved for nine therapeutic indications including, but not limited to, blepharospasm, strabismus, cervical dystonia (CD), focal spasticity including the treatment of upper limb spasticity associated with stroke, dynamic equinus foot deformity due to spasticity in pediatric cerebral palsy (CP), and primary hyperhidrosis of the axillae. ${ }^{6}$ Treatment paradigms with onabotulinumtoxinA vary greatly as recommendations on dose, dilution, and guidance techniques are specific to each indication. In addition to variability in recommended treatment paradigms, it is also known that treatment with onabotulinumtoxinA is individualized and often further varied in real-world clinical practice depending on disease presentation and desired outcomes. Although the highest recommended total adult dose of onabotulinumtoxinA in Canada is $360 \mathrm{U}$ and in the USA is $400 \mathrm{U}$ within a three-month period, reports have shown that doses exceeding this recommendation have been used in actual clinical practice. ${ }^{7,8}$ Therefore, it is very important to continue to monitor the safety profile of onabotulinumtoxinA in real-world use. ${ }^{9}$ Here we report on the safety and treatment parameters during a long-term, multicenter, observational trial, MOBILITY ${ }^{\circledR}$ (NCT00535935), using onabotulinumtoxinA for a variety of therapeutic indications in real-world clinical practice in Canada.

\section{Methods}

\section{Study Design and Population}

MOBILITY $^{\circledR}$ was a prospective, multicenter, observational Phase IV study conducted in Canada. Patients were enrolled into the study between October 2007 and July 2012 if treatment with onabotulinumtoxinA was determined to be medically necessary. Patients who were new to onabotulinumtoxinA treatment (naive) or those receiving ongoing treatments (maintenance) prior to study entry were included. Patients were consecutively approached, firstly to only the naive patients and then those on maintenance. Participants in the study were men and women $\geq 14$ years of age with no contraindications to use onabotulinumtoxin $\mathrm{A}$ and not participating in any onabotulinumtoxinA clinical trial. The safety cohort included all patients who were enrolled in the study and received at least one onabotulinumtoxinA treatment for their condition during the study.

Baseline clinical characteristics and demographics were collected at the initial visit. Safety and tolerability were monitored throughout the study period. Patients received their first onabotulinumtoxinA treatment at the baseline visit and were considered to have completed the study if they completed at least five subsequent treatments (six treatments total). Each participating physician was responsible for managing their own patient's condition (i.e., starting, stopping, or changing treatment) and for scheduling subsequent treatment visits, as treatments and treatment schedules were not stipulated in the study protocol. All data were collected on standardized case report forms and entered into an electronic database.

MOBILITY ${ }^{\circledR}$ was conducted in accordance with the principles of the Declaration of Helsinki and Good Clinical Practice, and the study protocol was approved by an independent ethics committee at each participating site. All patients were required to provide written informed consent prior to enrollment in the study.

\section{Study Measures}

Demographics and clinical characteristics were captured at the baseline visit. Adverse events (AEs) were documented at each subsequent treatment visit throughout the study. An AE was defined as any untoward medical occurrence that was temporally associated with onabotulinumtoxinA, regardless of causality. A serious AE (SAE) was defined as an AE that resulted in death, immediate risk of death ("life-threatening"), required or prolonged an existing hospitalization, persistent or significant disability, or was a congenital anomaly/birth defect. Physicians were required to report any $\mathrm{SAE}$ that occurred from the point that informed consent was obtained until 4 weeks after a patient ended study participation. Physicians were also required to report the degree to which an $\mathrm{AE}$ was related to onabotulinumtoxinA expressed as definite, probable, possible, unlikely, or unrelated. Additional treatment information, including dosing and injection frequency information, was collected following each treatment session.

\section{Statistical Analyses}

Patient demographics and onabotulinumtoxinA treatment information were summarized using descriptive statistics. The frequency and percent of all AEs were reported for the safety population, which included all patients who were enrolled in the study and received at least one onabotulinumtoxinA treatment during the study. Treatment-related AEs (TRAEs) include those reported by the investigator as possible, probable, highly probable, and related. Adverse events considered unrelated include those reported as unlikely and unrelated. MedDRA version 15.1 was used to summarize AEs by system organ class and preferred term. ${ }^{10}$ The analyses presented here were calculated using $\mathrm{SAS}^{\circledR}$ software, version 9.2 (SAS Institute Inc., Cary, NC, USA). There were no hypothesis testing or $P$-value calculations; however, $95 \%$ confidence intervals were provided around estimates of differences. As this project was exploratory in nature, no formal sample size calculations were carried out.

\section{RESULTS \\ Demographics}

A total of 1372 patients were enrolled into the study across 46 participating centers in Canada. All enrolled patients were included in the safety cohort. The majority of patients $(n=866 /$ $1372,63 \%$ ) were on maintenance onabotulinumtoxinA treatment upon study entry and had received between 1 and 90 treatments of onabotulinumtoxinA prior to entering the study (Table 1). Adult movement disorders (encompassing CD, blepharospasm, and hemifacial spasm [HFS], $n=455 / 1372$ ) and adult focal spasticity (AFS, $n=452 / 1372$ ) were the most commonly enrolled diagnoses (both 33\%), and cerebral palsy (CP) was the least common $(n=27 / 1372,2 \%)$. Due to differences in the management of adult versus pediatric $\mathrm{CP}$, adult $\mathrm{CP}$ patients were included in the AFS cohort while the CP cohort consisted of pediatric patients between 14 and 18 years old. A total of 150 enrolled patients were grouped under "other" diagnoses, which was mainly comprised of patients diagnosed with focal dystonia, headache, tremor, and pain. Of the 1372 enrolled patients, 519 (38\%) completed the five required subsequent visits as indicated in the study protocol, $149(11 \%)$ exited the study with less than 
Table 1: Patient characteristics stratified by indication and treatment history

\begin{tabular}{|c|c|c|c|c|c|c|c|c|c|c|c|c|c|c|c|}
\hline \multirow[b]{3}{*}{ Characteristic } & \multirow[b]{3}{*}{$\begin{array}{c}\text { Total } \\
N=\mathbf{1 3 7 2}\end{array}$} & \multicolumn{14}{|c|}{ Indication; no. (\%) patients ${ }^{\mathrm{a}}$} \\
\hline & & \multicolumn{2}{|c|}{ AFS } & \multicolumn{2}{|c|}{ Blepharospasm } & \multicolumn{2}{|c|}{ CP } & \multicolumn{2}{|c|}{ CD } & \multicolumn{2}{|c|}{ HFS } & \multicolumn{2}{|c|}{ HH } & \multicolumn{2}{|c|}{ Other } \\
\hline & & $\begin{array}{c}\text { naive } \\
n=178\end{array}$ & $\begin{array}{c}\text { maint } \\
n=274\end{array}$ & $\begin{array}{c}\text { naive } \\
n=24\end{array}$ & $\begin{array}{l}\text { maint } \\
n=59\end{array}$ & $\begin{array}{l}\text { naive } \\
n=5\end{array}$ & $\begin{array}{l}\text { maint } \\
n=22\end{array}$ & $\begin{array}{c}\text { naive } \\
n=48\end{array}$ & $\begin{array}{c}\text { maint } \\
n=202\end{array}$ & $\begin{array}{c}\text { naive } \\
n=42\end{array}$ & $\begin{array}{l}\text { maint } \\
n=80\end{array}$ & $\begin{array}{c}\text { naive } \\
n=151\end{array}$ & $\begin{array}{c}\text { maint } \\
n=137\end{array}$ & $\begin{array}{c}\text { naive } \\
n=58\end{array}$ & $\begin{array}{l}\text { maint } \\
n=92\end{array}$ \\
\hline Age, years & $50 \pm 18$ & $54 \pm 15$ & $52 \pm 17$ & $68 \pm 13$ & $61 \pm 11$ & $16 \pm 1$ & $16 \pm 1$ & $55 \pm 15$ & $58 \pm 11$ & $60 \pm 13$ & $67 \pm 11$ & $32 \pm 12$ & $33 \pm 11$ & $56 \pm 15$ & $52 \pm 12$ \\
\hline Diff $(95 \% \mathrm{CI})^{\mathrm{b}}$ & $-2.6(-4.6,-0.7)$ & \multicolumn{2}{|c|}{$1.9(-1.1,4.9)$} & \multicolumn{2}{|c|}{$6.8(0.8,12.8)$} & \multicolumn{2}{|c|}{$0.4(-1.3,2.2)$} & \multicolumn{2}{|c|}{$-2.1(-6.7,2.4)$} & \multicolumn{2}{|c|}{$-6.2(-10.8,-1.5)$} & \multicolumn{2}{|c|}{$-0.5(-3.1,2.2)$} & \multicolumn{2}{|c|}{$4.3(-0.3,9.0)$} \\
\hline $14-17$ & $50(4)$ & $0(0)$ & $0(0)$ & $0(0)$ & $0(0)$ & $5(100)$ & $22(100)$ & $1(2)$ & $1(1)$ & $0(0)$ & $0(0)$ & $16(11)$ & $5(4)$ & $0(0)$ & $0(0)$ \\
\hline $18-65$ & $1015(74)$ & $135(76)$ & $208(76)$ & $10(42)$ & $37(63)$ & $0(0)$ & $0(0)$ & $36(75)$ & $149(74)$ & $22(52)$ & $35(44)$ & $133(88)$ & $129(94)$ & $38(66)$ & $83(90)$ \\
\hline$\geq 65$ & $307(22)$ & $43(24)$ & $66(24)$ & $14(58)$ & $22(37)$ & $0(0)$ & $0(0)$ & $11(23)$ & $52(26)$ & $20(48)$ & $45(56)$ & $2(1)$ & $3(2)$ & $20(35)$ & $9(10)$ \\
\hline \multicolumn{16}{|l|}{ Gender } \\
\hline Female & $881(64)$ & $77(43)$ & $152(56)$ & $13(54)$ & $37(63)$ & $2(40)$ & $8(36)$ & $35(73)$ & $154(76)$ & $28(67)$ & $52(65)$ & $109(72)$ & $113(83)$ & $40(69)$ & $61(66)$ \\
\hline Male & $491(36)$ & $101(57)$ & $122(45)$ & $11(46)$ & $22(37)$ & $3(60)$ & $14(64)$ & $13(27)$ & $48(24)$ & $14(33)$ & $28(35)$ & $42(28)$ & $24(18)$ & $18(31)$ & $31(34)$ \\
\hline \multicolumn{16}{|l|}{ Race } \\
\hline White/Caucasian & $1253(91)$ & $156(88)$ & $156(88)$ & $22(92)$ & $57(97)$ & $4(80)$ & $17(77)$ & $46(96)$ & $196(97)$ & $36(86)$ & 79 (95) & $137(91)$ & $128(93)$ & $56(97)$ & $86(94)$ \\
\hline Other $^{c}$ & $119(9)$ & $22(12)$ & $118(43)$ & $2(8)$ & $2(3)$ & $1(20)$ & $5(23)$ & $2(4)$ & $6(3)$ & $6(14)$ & $1(1)$ & $12(9)$ & $9(7)$ & $2(3)$ & $6(7)$ \\
\hline Weight, $\mathrm{kg}$, mean $\pm \mathrm{SD}$ & $73 \pm 17$ & $73 \pm 16$ & $74 \pm 18$ & $77 \pm 17$ & $74 \pm 16$ & $48 \pm 27$ & $45 \pm 13$ & $72 \pm 15$ & $74 \pm 17$ & $77 \pm 18$ & $73 \pm 14$ & $70 \pm 14$ & $70 \pm 15$ & $76 \pm 16$ & $77 \pm 17$ \\
\hline Height, $\mathrm{cm}$, mean $\pm \mathrm{SD}$ & $166 \pm 11$ & $168 \pm 11$ & $166 \pm 11$ & $167 \pm 10$ & $166 \pm 10$ & $163 \pm 23$ & $164 \pm 9$ & $165 \pm 12$ & $166 \pm 9$ & $165 \pm 10$ & $165 \pm 10$ & $167 \pm 11$ & $167 \pm 12$ & $166 \pm 12$ & $167 \pm 10$ \\
\hline BMI, $\mathrm{kg} / \mathrm{m}^{2}$, mean $\pm \mathrm{SD}$ & $26 \pm 6$ & $26 \pm 5$ & $27 \pm 7$ & $28 \pm 4$ & $27 \pm 5$ & $21 \pm 6$ & $19 \pm 2$ & $26 \pm 5$ & $27 \pm 6$ & $28 \pm 6$ & $27 \pm 5$ & $25 \pm 5$ & $25 \pm 5$ & $28 \pm 6$ & $28 \pm 6$ \\
\hline \multicolumn{16}{|c|}{ Total treatments, prior to study ${ }^{\mathrm{d}}$} \\
\hline Mean \pm SD & $11 \pm 13$ & NA & $6 \pm 6$ & NA & $20 \pm 18$ & NA & $6 \pm 5$ & NA & $17 \pm 16$ & NA & $19 \pm 18$ & NA & $3 \pm 2$ & NA & $11 \pm 13$ \\
\hline Median & 6 & & 5 & & 11 & & 4 & & 12 & & 14 & & 2 & & 6.5 \\
\hline Range & $1-90$ & & $1-32$ & & $1-68$ & & $1-19$ & & $1-90$ & & $1-80$ & & $1-9$ & & $1-52$ \\
\hline Dose, units mean & 221 & 285 & 350 & 47.4 & 62.9 & 250 & 253 & 173 & 248 & 32.6 & 42.2 & 193 & 204 & 173 & 192 \\
\hline SD & 148 & 147 & 149 & 20.0 & 44.6 & 84 & 105 & 71 & 102 & 14.8 & 27.6 & 63 & 77 & 103 & 114 \\
\hline Median & 200 & 300 & 300 & 50.0 & 50.0 & 200 & 300 & 180 & 200 & 35.0 & 40.0 & 200 & 200 & 140 & 200 \\
\hline Range & 3-900 & $20-800$ & 30-900 & $15-100$ & $5.5-350$ & $200-400$ & $100-400$ & $4-400$ & $4-700$ & $5-100$ & $5-200$ & $75-500$ & $25-500$ & $20-600$ & $3-500$ \\
\hline
\end{tabular}

$\mathrm{AFS}=$ adult focal spasticity; $\mathrm{BMI}=$ body mass index; $\mathrm{CD}=$ cervical dystonia; $\mathrm{CI}=$ confidence interval; $\mathrm{CP}=$ cerebral palsy; Diff = difference; $\mathrm{HFS}=$ hemifacial spasm; $\mathrm{HH}=$ hyperhidrosis; maint $=$ maintenance; $\mathrm{NA}=$ not applicable; $\mathrm{SD}=$ standard deviation .

${ }^{a}$ Unless otherwise indicated.

${ }^{\mathrm{b}}$ Difference calculated for naive minus maintenance groups, for means.

'Includes the following that were each $\leq 5 \%$ of the population: Aboriginal, Arab/West Asian, Black, Chinese, Filipino, Japanese, Korean, Latin American, South Asian, Southeast Asian, and/or those

categorized as "other," except for the CP cohort where South Asian accounted for 9\% $(n=2)$ of the maintenance population.

${ }^{\mathrm{d}}$ Number of onabotulinumtoxinA injections received prior to entering the study; maintenance patients only. 
(A)

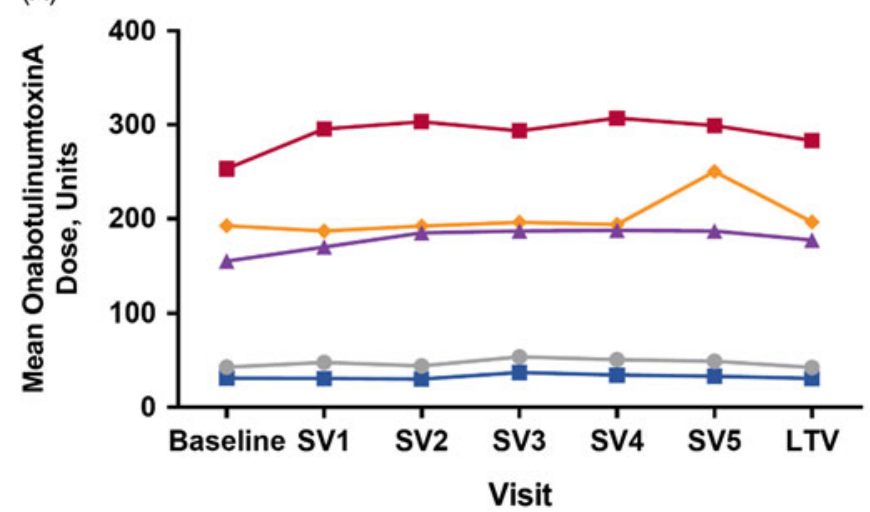

(B)

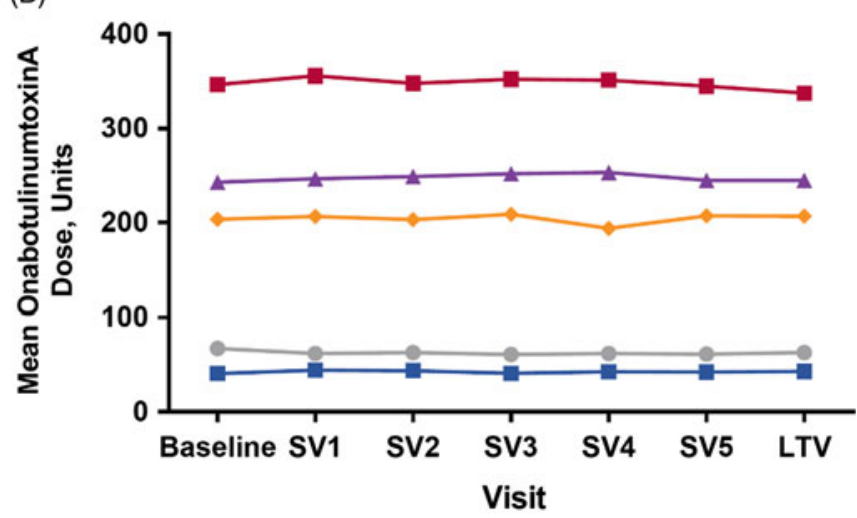

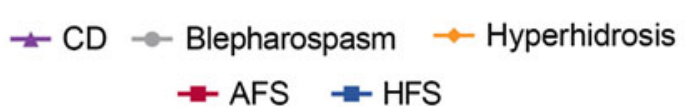

Figure 1: Mean administered dose of onabotulinumtoxinA at each treatment visit for (A) naive and (B) maintenance patients. Note: Plot for cerebral palsy cohort not presented due to low patient number; plot for "other" diagnoses not included due to heterogeneity of indications. $A F S=$ adult focal spasticity, CD = cervical dystonia, HFS = hemifacial spasm, SV = subsequent visit, LTV $=$ last treatment visit.

the five subsequent visits, and 704 (51\%) discontinued the study. Of the patients who discontinued, the proportions of naive $(n=336 / 704,48 \%)$ and maintenance $(n=368 / 704,52 \%)$ patients were comparable. The majority of the patients who discontinued the study were due to reasons reported as "other" $(n=363 / 704,52 \%)$, lost to follow-up $(n=195 / 704,28 \%)$, and patient withdrawing consent $(n=92 / 704,13 \%)$. Study discontinuations grouped under "other" included study site closure, investigator study discontinuation, limited availability of resources (i.e., clinic nurse) at the participating clinic, or subject follow-up visits not carried out. Only 6 out of 704 of the discontinued patients $(0.9 \%)$ were due to AEs, comprising 2 $(0.6 \%)$ naive and $4(1.1 \%)$ maintenance patients. Among the six patients, only two discontinued the study due to a TRAE (facial paresis/eyelid ptosis and dysphagia).Those who discontinued due to lack of drug efficacy only accounted for $4 \%(n=31 / 704)$ of the discontinued patient population. Reasons for study discontinuation were similar between naive and maintenance patients and between indications. Patient demographics exhibited some variability across indications, consistent with the range of therapeutic indications treated in this study. The oldest patient population consisted of those who were diagnosed with HFS, whereas the youngest population was of patients with $\mathrm{CP}$ (Table 1). Consistent with the youngest population, $\mathrm{CP}$ patients reported the lowest weight, height, and body mass index at baseline compared to other indications. However, gender and race distributions were generally comparable between indications with the majority of patients being female $(n=881 / 1372,64 \%)$ and Caucasian $(n=1253 / 1372,91 \%)$. Within the maintenance population, HFS, $\mathrm{CD}$, and blepharospasm patients received the most number of onabotulinumtoxinA treatments prior to study entry (1-90 treatments), whereas patients with AFS, CP, and hyperhidrosis received the fewest (1-32 treatments).

The mean (standard deviation) and median doses of onabotulinumtoxinA were $221 \mathrm{U}$ (148). Naive patients generally received lower doses when compared to maintenance, and doses varied widely by indication. For example, $\mathrm{CD}$ naive patients received a mean (SD) dose of $173 \mathrm{U}(71)$, whereas maintenance patients received a mean (SD) dose of $248 \mathrm{U}$ (102) (Table 1). Patients who presented with AFS received the highest average (SD) doses (naive: $285 \mathrm{U}$ [147], maintenance: $350 \mathrm{U}$ [149]) during the study, whereas HFS patients received the lowest average (SD) doses (naive: $32.6 \mathrm{U}$ [14.8], maintenance: 42.2 U [27.6]). Dosing remained consistent throughout the study within each indication for treatment naive (Figure 1A) and maintenance (Figure 1B) patients.

\section{Safety}

A total of 209 AEs were reported in 83 patients $(83 / 1372,6 \%)$ in the overall safety cohort (Table 2). Of the 209 AEs, 72 were serious AEs reported in 38 patients $(38 / 1372,3 \%)$. The majority of reported AEs $(143 / 209,68 \%)$ were determined to be unrelated to treatment by the participating investigator. The causalities for 22 reported AEs $(22 / 209,11 \%)$ were not determined or were not reported. Forty-four AEs $(44 / 209,21 \%)$ in 24 patients were reported as TRAEs, of which 10 AEs in 5 patients were reported as being a serious TRAE. Of the 24 patients who reported TRAEs, only $2(2 / 24,8 \%)$ patients, representing only $0.15 \%$ of the entire safety cohort, discontinued the study (facial paresis/ eyelid ptosis and dysphagia).

Among the safety cohort $(n=1372), 13$ patients $(0.9 \%)$ who were naive to onabotulinumtoxinA treatment reported 24/44 TRAEs, whereas $11(0.8 \%)$ maintenance patients had 20/44 TRAEs, as determined by the investigator. The most commonly reported TRAEs across indications were muscular weakness $(n=8 / 1372,0.6 \%)$ and dysphagia $(n=6 / 1372,0.4 \%)$. The majority of TRAEs were reported in patients presenting with "other" indications (Table 3 ). A total of six patients $(0.4 \%)$ categorized as "other" diagnoses reported 13/44 TRAEs, as assessed by the investigator. Of these six "other" patients, with a diagnosis of focal dystonia $(n=3)$, headache $(n=1)$, pain $(n=1)$, and tremor $(n=1)$, three commonly reported muscular weakness as an AE. No AEs were reported in the CP cohort. There was one TRAE (herpes zoster in an AFS patient) that was considered to be related to the injection procedure. A total of 14 deaths were reported in the safety cohort, and none were considered treatment related by 
Table 2: Characteristics of all reported adverse events by indication and treatment history

\begin{tabular}{|c|c|c|c|c|c|c|c|c|c|c|c|c|c|}
\hline \multirow[b]{3}{*}{ Adverse Event Category ${ }^{a}$} & \multirow[b]{3}{*}{$\begin{array}{c}\text { Total } \\
N=1372\end{array}$} & \multicolumn{12}{|c|}{ Indication; no. (\%) patients } \\
\hline & & \multicolumn{2}{|c|}{ AFS } & \multicolumn{2}{|c|}{ Blepharospasm } & \multicolumn{2}{|c|}{ CD } & \multicolumn{2}{|c|}{ HFS } & \multicolumn{2}{|c|}{ HH } & \multicolumn{2}{|c|}{ Other } \\
\hline & & $\begin{array}{c}\text { naive } \\
n=178\end{array}$ & $\begin{array}{c}\text { maint } \\
n=274\end{array}$ & $\begin{array}{c}\text { naive } \\
n=24\end{array}$ & $\begin{array}{l}\text { maint } \\
n=59\end{array}$ & $\begin{array}{c}\text { naive } \\
n=48\end{array}$ & $\begin{array}{c}\text { maint } \\
n=202\end{array}$ & $\begin{array}{c}\text { naive } \\
n=42\end{array}$ & $\begin{array}{l}\text { maint } \\
n=80\end{array}$ & $\begin{array}{c}\text { naive } \\
n=151\end{array}$ & $\begin{array}{c}\text { maint } \\
n=137\end{array}$ & $\begin{array}{c}\text { naive } \\
n=58\end{array}$ & $\begin{array}{l}\text { maint } \\
n=92\end{array}$ \\
\hline All AEs & $83(6)$ & $9(5)$ & $12(4)$ & $3(13)$ & $2(3)$ & $3(6)$ & $30(15)$ & $2(5)$ & 7 (9) & $1(<1)$ & $0(0)$ & $4(7)$ & $10(11)$ \\
\hline Treatment-related & 24 (2) & $2(1)$ & $2(1)$ & $2(8)$ & $2(3)$ & $3(6)$ & $3(1)$ & $2(5)$ & $1(1)$ & $1(<1)$ & $0(0)$ & $3(5)$ & $3(3)$ \\
\hline Treatment-unrelated & 55 (4) & $5(3)$ & 7 (3) & $1(4)$ & $2(3)$ & $2(4)$ & $25(12)$ & $0(0)$ & 7 (9) & $0(0)$ & $0(0)$ & $2(3)$ & $7(8)$ \\
\hline Unknown / Not reported & $12(1)$ & $1(<1)$ & $4(1)$ & $0(0)$ & $0(0)$ & $0(0)$ & $6(3)$ & $0(0)$ & $0(0)$ & $1(<1)$ & $0(0)$ & $0(0)$ & $0(0)$ \\
\hline All serious AEs & $38(3)$ & $4(2)$ & $8(3)$ & $0(0)$ & $1(2)$ & $0(0)$ & $17(8)$ & $0(0)$ & $4(5)$ & $0(0)$ & $0(0)$ & $1(2)$ & $3(3)$ \\
\hline Treatment-related & $5(<1)$ & $0(0)$ & $0(0)$ & $0(0)$ & $1(2)$ & $0(0)$ & $3(1)$ & $0(0)$ & $0(0)$ & $0(0)$ & $0(0)$ & $1(2)$ & $0(0)$ \\
\hline Treatment-unrelated & $27(2)$ & $3(2)$ & $6(2)$ & $0(0)$ & $0(0)$ & $0(0)$ & $13(6)$ & $0(0)$ & $4(5)$ & $0(0)$ & $0(0)$ & $0(0)$ & $3(3)$ \\
\hline Unknown/not reported & $12(1)$ & $1(<1)$ & $4(1)$ & $0(0)$ & $0(0)$ & $0(0)$ & $6(3)$ & $0(0)$ & $0(0)$ & $1(<1)$ & $0(0)$ & $0(0)$ & $0(0)$ \\
\hline Discontinued due to $\mathrm{AE}$ & $6(<1)$ & $1(<1)$ & $1(<1)$ & $0(0)$ & $0(0)$ & $0(0)$ & $2(1)$ & $0(0)$ & $0(0)$ & $1(<1)$ & $0(0)$ & $0(0)$ & $1(1)$ \\
\hline Treatment-related & $2(<1)$ & $0(0)$ & $0(0)$ & $0(0)$ & $0(0)$ & $0(0)$ & $1(<1)$ & $0(0)$ & $0(0)$ & $1(<1)$ & $0(0)$ & $0(0)$ & $0(0)$ \\
\hline Treatment-unrelated & $3(<1)$ & $1(<1)$ & $0(0)$ & $0(0)$ & $0(0)$ & $0(0)$ & $1(<1)$ & $0(0)$ & $0(0)$ & $0(0)$ & $0(0)$ & $0(0)$ & $1(1)$ \\
\hline Unknown/not reported & $2(<1)$ & $0(0)$ & $1(<1)$ & $0(0)$ & $0(0)$ & $0(0)$ & $0(0)$ & $0(0)$ & $0(0)$ & $1(<1)$ & $0(0)$ & $0(0)$ & $0(0)$ \\
\hline Death & $14(1)$ & $2(1)$ & $7(3)$ & $0(0)$ & $0(0)$ & $0(0)$ & $2(1)$ & $0(0)$ & $1(1)$ & $0(0)$ & $0(0)$ & $0(0)$ & $2(2)$ \\
\hline Treatment-related & $0(0)$ & $0(0)$ & $0(0)$ & $0(0)$ & $0(0)$ & $0(0)$ & $0(0)$ & $0(0)$ & $0(0)$ & $0(0)$ & $0(0)$ & $0(0)$ & $0(0)$ \\
\hline Treatment-unrelated & $11(1)$ & $2(1)$ & $5(2)$ & $0(0)$ & $0(0)$ & $0(0)$ & $1(1)$ & $0(0)$ & $1(1)$ & $0(0)$ & $0(0)$ & $0(0)$ & $2(2)$ \\
\hline Unknown/not reported & $2(<1)$ & $0(0)$ & $2(1)$ & $0(0)$ & $0(0)$ & $0(0)$ & $0(0)$ & $0(0)$ & $0(0)$ & $0(0)$ & $0(0)$ & $0(0)$ & $0(0)$ \\
\hline Total number of reported $\mathrm{AEs}^{\mathrm{b}}$ & 209 & \multicolumn{2}{|c|}{36} & \multicolumn{2}{|c|}{20} & \multicolumn{2}{|c|}{95} & \multicolumn{2}{|c|}{21} & \multicolumn{2}{|c|}{3} & \multicolumn{2}{|c|}{3} \\
\hline
\end{tabular}

$\mathrm{AE}=$ adverse event; $\mathrm{AFS}=$ adult focal spasticity; $\mathrm{BMI}=$ body mass index; $\mathrm{CD}=$ cervical dystonia $\mathrm{CI}=$ confidence interval; $\mathrm{CP}=$ cerebral palsy; Diff $=$ difference; $\mathrm{HFS}=$ hemifacial spasm; $\mathrm{HH}=$ hyperhidrosis; maint $=$ maintenance .

No AEs were reported in the CP population. Treatment-related AEs include those reported as "related," "highly probable," "probable," and "possible" by the investigator; treatment-unrelated AEs include those reported as "Not related" and "unlikely" by the investigator.

${ }^{a}$ Each patient is only counted once for each AE category.

${ }^{\mathrm{b}}$ Total number of of AEs reported across all patients. Each patient may be counted more than once. 
Table 3: Listing of all treatment-related adverse events by indication as reported by the investigator

\begin{tabular}{|c|c|c|c|c|c|c|c|c|c|c|c|c|c|}
\hline \multirow[b]{3}{*}{$\begin{array}{l}\text { Adverse event by } \\
\text { preferred term }^{\mathbf{a}}\end{array}$} & \multirow[b]{3}{*}{$\begin{array}{c}\text { Total } \\
N=1372\end{array}$} & \multicolumn{12}{|c|}{ Indication } \\
\hline & & \multicolumn{2}{|c|}{ AFS } & \multicolumn{2}{|c|}{ Blepharospasm } & \multicolumn{2}{|c|}{ CD } & \multicolumn{2}{|c|}{ HFS } & \multicolumn{2}{|c|}{ HH } & \multicolumn{2}{|c|}{ Other } \\
\hline & & $\begin{array}{c}\text { naive } \\
n=178\end{array}$ & $\begin{array}{c}\text { maint } \\
n=274\end{array}$ & $\begin{array}{c}\text { naive } \\
n=24\end{array}$ & $\begin{array}{l}\text { maint } \\
n=59\end{array}$ & $\begin{array}{c}\text { naive } \\
n=48\end{array}$ & $\begin{array}{c}\text { maint } \\
n=202\end{array}$ & $\begin{array}{c}\text { naive } \\
n=42\end{array}$ & $\begin{array}{l}\text { maint } \\
n=80\end{array}$ & $\begin{array}{c}\text { naive } \\
n=151\end{array}$ & $\begin{array}{c}\text { maint } \\
n=137\end{array}$ & $\begin{array}{c}\text { naive } \\
n=58\end{array}$ & $\begin{array}{l}\text { maint } \\
n=92\end{array}$ \\
\hline Overall, $n(\%)$ & $42(3)$ & $4(2)$ & $3(1)$ & $2(8)$ & $4(7)$ & $4(8)$ & $8(4)$ & $2(5)$ & $1(1)$ & $1(1)$ & $0(0)$ & $10(17)$ & $3(3)$ \\
\hline Muscular weakness & 7 & 1 & - & - & - & 2 & 1 & - & - & - & - & - & 3 \\
\hline Dysphagia & 6 & - & - & - & 1 & 1 & 3 & - & - & - & - & 1 & - \\
\hline Headache & 3 & - & - & - & - & 1 & 1 & - & - & - & - & 1 & - \\
\hline Eyelid ptosis & 2 & - & - & 1 & - & - & - & - & - & - & - & 1 & - \\
\hline Facial paresis & 2 & - & - & - & - & - & - & 1 & - & 1 & - & - & - \\
\hline Lacrimation increased & 2 & - & - & - & - & - & - & - & 1 & - & - & 1 & - \\
\hline Local swelling & 2 & - & 1 & - & - & - & 1 & - & - & - & - & - & - \\
\hline Asthenia & 1 & - & - & - & - & - & - & - & - & - & - & 1 & - \\
\hline Blepharochalasis & 1 & - & - & - & 1 & - & - & - & - & - & - & - & - \\
\hline Choking & 1 & - & - & - & 1 & - & - & - & - & - & - & - & - \\
\hline Diarrhea & 1 & - & - & - & - & - & - & - & - & - & - & 1 & - \\
\hline Dysphonia & 1 & - & - & - & 1 & - & - & - & - & - & - & - & - \\
\hline Herpes zoster & 1 & - & 1 & - & - & - & - & - & - & - & - & - & - \\
\hline Injection site bruising & 1 & - & - & 1 & - & - & - & - & - & - & - & - & - \\
\hline Joint stiffness & 1 & 1 & - & - & - & - & - & - & - & - & - & - & - \\
\hline Muscle tightness & 1 & - & - & - & - & - & 1 & - & - & - & - & - & - \\
\hline $\begin{array}{l}\text { Musculoskeletal } \\
\text { stiffness }\end{array}$ & 1 & 1 & - & - & - & - & - & - & - & - & - & - & - \\
\hline Nausea & 1 & - & - & - & - & - & - & - & - & - & - & 1 & - \\
\hline Pain & 1 & - & - & - & - & - & - & - & - & - & - & 1 & - \\
\hline Pain in extremity & 1 & - & 1 & - & - & - & - & - & - & - & - & - & - \\
\hline Pyrexia & 1 & - & - & - & - & - & - & - & - & - & - & 1 & - \\
\hline Swelling face & 1 & - & - & - & - & - & - & 1 & - & - & - & - & - \\
\hline Tremor & 1 & - & - & - & - & - & - & - & - & - & - & 1 & - \\
\hline Urticaria & 1 & 1 & - & - & - & - & - & - & - & - & - & - & - \\
\hline
\end{tabular}

$\mathrm{AFS}=$ adult focal spasticity; $\mathrm{CD}=$ cervical dystonia; $\mathrm{CP}=$ cerebral palsy; $\mathrm{HFS}=$ hemifacial spasm; $\mathrm{HH}=$ hyperhidrosis; maint $=$ maintenance.

No treatment-related AEs were reported in the CP population. Treatment-related AEs include those reported as "related," "highly probable," "probable," and "possible" by the investigator.

${ }^{\text {a }}$ Patients counted once for each listed AE when multiple occurrences were reported. One patient with AFS reported two muscular weakness AEs, and one patient with blepharospasm reported two dysphonia adverse events; however, each was only counted once here.

the investigator. There were no pregnancies or abortions reported during the study.

Although the majority of patients $(n=888 / 1372,65 \%)$ received a baseline dose of $\geq 200 \mathrm{U}$, the frequency of TRAEs was highest in patients administered $\leq 199 \mathrm{U}$ at baseline (Figure 2). There was no statistically significant association between the incidence of TRAEs and the dose of onabotulinumtoxinA at baseline (Mantel Haenszel Chi-square test, $P=0.0237$; Figure 2). When compared across age groups, incidence of TRAEs tended to increase with increasing age; there was a statistically significant association between the incidence of TRAEs and the patient's age group (Mantel Haenszel Chi-square test, $P=0.002$; Figure 3).

\section{Adult Focal Spasticity}

Of 452 AFS patients, 17 patients reported 33 AEs (17/452, $4 \%$ ), of which $8 \mathrm{AEs}$ in four patients $(4 / 452,0.9 \%)$ were considered TRAEs by the investigator. No single AE was reported by more than one AFS patient. The reported TRAEs were equally distributed between naive and maintenance patients, and no apparent trends were observed with regard to treatment pattern. Two patients who received injections of onabotulinumtoxinA $400 \mathrm{U}$ and $500 \mathrm{U}$, which included injections in the hand, experienced TRAEs of weakness and stiffness in the hand and finger and swelling and pain in the hand that was suggestive of complex regional pain syndrome, respectively. 


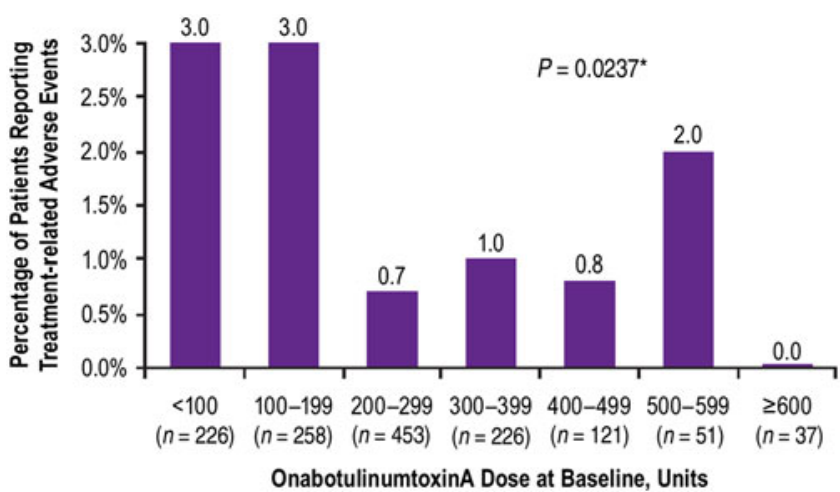

Figure 2: Percent of patients who reported treatment-related adverse events by dose administered at baseline. *Mantel Haenzel Chi-square test for association between treatment-related adverse events and onabotulinumtoxinA dose at baseline.

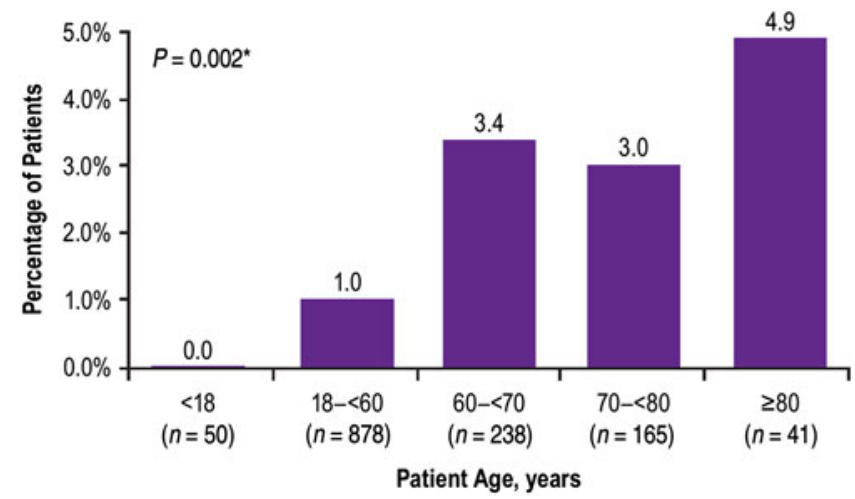

Figure 3: Percent of patients who reported treatment-related adverse events by age at baseline. *Mantel Haenzel Chi-square test for association between treatment-related adverse events and patient's age group.

AFS patients did not report any serious TRAEs. Out of a total of 14 deaths, nine were reported in the AFS cohort, of which four with known etiology were determined to be unrelated to treatment by the investigator (progression of multiple sclerosis, malignant neoplasm, sudden cardiac death, and complications from cardiomegaly/diabetes/pulmonary edema). The cause of death for $4 / 9$ of the fatalities was not specified by the investigators, although for 3 of these patients the investigators did report that the deaths were either not (2 patients), or were unlikely to be (1 patient), related to the study treatment. For the remaining patient (a 71-year-old male), no information on relation to treatment was reported. No serious bleeding events were reported. There were no discontinuations due to TRAEs.

\section{Pediatric Cerebral Palsy}

There were no reported AEs in the 27 patients with diagnosed $\mathrm{CP}$ who received onabotulinumtoxinA treatments in the study.

\section{Movement Disorders}

Of the 455 patients with blepharospasm $(n=83), \mathrm{CD}$ $(n=250)$, or HFS $(n=122)$, a total of 136 AEs were reported by 46 (10\%; Table 2). Within the blepharospasm and HFS cohorts $(n=205)$, where facial muscles were injected, seven patients $(3.4 \%)$ reported a total of 10 TRAEs. Each TRAE was reported by only one patient. There were no major trends in reported TRAEs with respect to treatment history or treatment characteristics.

One serious AE (dysphagia) possibly related to treatment was reported in one maintenance dystonia patient with Meige's syndrome. The patient received six intramuscular treatments of onabotulinumtoxinA between $147.5 \mathrm{U}$ and $150 \mathrm{U}$ for the maintenance indication, including for blepharospasm and spasmodic dysphonia (the latter being outside of the MOBILITY study; Table 4). One death due to small bowel obstruction in a patient with facial nerve disorder was reported as not related to treatment by the investigator.

In the CD group $(n=250)$, a total of 95 AEs were reported by $33(13 \%)$ patients (Table 2). In six (2.4\%) patients, $12(13 \%)$ of the 95 AEs reported were considered TRAEs by the investigator. More than one TRAE could be reported by a patient, and the most commonly reported TRAEs in the $\mathrm{CD}$ group were dysphagia $(n=4 / 250,2 \%)$ and muscular weakness $(n=3 / 250,1 \%)$. There did not appear to be any correlation between TRAEs and treatment history or treatment characteristics. A total of eight serious TRAEs were reported in three patients (1\%). All patients who reported serious TRAEs were on maintenance onabotulinumtoxinA treatment. One patient with a serious TRAE (dysphagia) subsequently discontinued the study. Two fatal cases were reported, due to bowel infarction and metastases to lung, and both were considered unrelated to treatment.

\section{Hyperhidrosis}

Of the 288 patients in the hyperhidrosis cohort, 1 patient $(<1 \%)$ treated for facial sweating reported a total of three AEs (eyelid ptosis, worsening hyperhidrosis, and facial paresis). The mild facial paresis and eyelid ptosis were the only AEs considered treatment related and appeared to be local effects, likely related to the location of the injection. Both are well-known temporary side effects of this treatment. This patient withdrew from the study.

\section{Other Diagnoses}

By definition, in this study patients categorized under "other" diagnoses $(n=150)$ as classified by investigators presented with focal dystonia, headache, pain, tics, tremors, or multiple indications. These patients reported a total of 33 AEs in 14 out of 150 patients (9\%) (Table 2). Of the 33 AEs reported, 13 AEs in 6 out of 150 patients $(4 \%)$ were reported as TRAEs by the investigator. Muscular weakness was the most commonly reported TRAE (7/44, 17\%), which was reported by three patients in the maintenance cohort (Table 3). There did not appear to be any major trend in TRAEs with regard to treatment history or treatment characteristics. One serious TRAE (dysphagia) was reported in one patient who had presented with focal dystonia. Two deaths were reported as unrelated to treatment. One death, due to myocardial infarction, was reported in one patient who had been treated with onabotulinumtoxinA for pain. The second death, due to Parkinson's disease, was reported in a patient who had been treated for oromandibular dystonia.

\section{Discussion}

The purpose of MOBILITY was to examine the long-term safety of onabotulinumtoxinA following multiple treatments 
Table 4: Characteristics of investigator-reported, treatment-related adverse events

\begin{tabular}{|c|c|c|c|c|c|c|c|c|}
\hline $\begin{array}{l}\text { Adverse event by } \\
\text { indication }^{\text {a }}\end{array}$ & Patient $^{\mathrm{a}}$ & Age & Treatment history & Serious event & $\begin{array}{c}\text { Symptom } \\
\text { duration (days) }\end{array}$ & No. injections $^{b}$ & Dose $^{c}$ (units) & Dilution ratio \\
\hline \multicolumn{9}{|c|}{ Adult focal spasticity } \\
\hline Herpes zoster & 1 & 60 & maint & no & 34 & 6 & $250-325$ & $2-2.3$ \\
\hline Joint stiffness & 2 & 27 & naive & no & 92 & 1 & 400 & 2 \\
\hline $\begin{array}{l}\text { Muscular } \\
\text { weakness }\end{array}$ & 2 & 27 & naive & no & 92 & 1 & 400 & 2 \\
\hline $\begin{array}{l}\text { Musculoskeletal } \\
\text { stiffness }\end{array}$ & 2 & 27 & naive & no & 92 & 1 & 400 & 2 \\
\hline Local swelling & 3 & 53 & maint & no & ongoing $^{\mathrm{e}}$ & 1 & 400 & 2 \\
\hline Pain in extremity & 3 & 53 & maint & no & ongoing $^{\mathrm{e}}$ & 1 & 500 & 2 \\
\hline Urticaria & 4 & 60 & naive & no & 21 & 1 & 100 & 2 \\
\hline \multicolumn{9}{|c|}{ Blepharospasm } \\
\hline Blepharochalasis & 5 & 79 & maint & no & 98 & 2 & $40-125$ & 1 \\
\hline Choking & $6^{\mathrm{f}}$ & 64 & maint & no & NR & 6 & $147.5-150$ & $0.9-1$ \\
\hline Dysphagia & $6^{\mathrm{f}}$ & 64 & maint & yes & NR & 6 & $147.5-150$ & $0.9-1$ \\
\hline Dysphonia & $6^{\mathrm{f}}$ & 64 & maint & no & $\mathrm{NR}^{\mathrm{g}}$ & 6 & $147.5-150$ & $0.9-1$ \\
\hline Eyelid ptosis & 7 & 56 & naive & no & ongoing $^{\mathrm{e}}$ & 2 & 25 & 1 \\
\hline $\begin{array}{l}\text { Injection site } \\
\text { bruising }\end{array}$ & 8 & 54 & naive & no & 14 & 2 & 15 & 2 \\
\hline \multicolumn{9}{|c|}{ Cervical dystonia } \\
\hline \multirow[t]{4}{*}{ Dysphagia } & 9 & 81 & maint & yes & NA & 1 & 200 & 1.2 \\
\hline & 10 & 67 & maint & yes & NR & 4 & $300-320$ & 1 \\
\hline & 11 & 77 & maint & yes & 42 & 3 & 350 & 1 \\
\hline & 12 & 45 & naive & no & 52 & 1 & 150 & 1 \\
\hline \multirow[t]{2}{*}{ Headache } & 11 & 77 & maint & yes & 42 & 3 & 350 & 1 \\
\hline & 13 & 59 & naive & no & 21 & 1 & 100 & 1 \\
\hline Local swelling & 11 & 77 & maint & yes & NR & 3 & 350 & 1 \\
\hline Muscle tightness & 10 & 67 & maint & yes & ongoing $^{\mathrm{e}}$ & 4 & $300-320$ & 1 \\
\hline \multirow{3}{*}{$\begin{array}{l}\text { Muscular } \\
\text { weakness }\end{array}$} & 10 & 67 & maint & yes & NR & 4 & $300-320$ & 1 \\
\hline & 14 & 48 & naive & no & NR & 4 & $150-275$ & 1 \\
\hline & 13 & 59 & naive & no & NR & 1 & 100 & 1 \\
\hline \multicolumn{9}{|c|}{ Hemifacial spasm } \\
\hline Facial paresis & 15 & 66 & naive & no & 66 & 1 & 20 & 0.2 \\
\hline $\begin{array}{c}\text { Lacrimation } \\
\text { increased }\end{array}$ & 16 & 63 & maint & no & 15 & 6 & $15-20$ & 2 \\
\hline Swelling face & 17 & 70 & naive & no & ongoing $^{\mathrm{e}}$ & 1 & 20 & 1 \\
\hline \multicolumn{9}{|c|}{ Hyperhidrosis } \\
\hline Facial paresis & 18 & 53 & naive & no & 117 & 1 & 100 & 3 \\
\hline \multicolumn{9}{|c|}{ Other } \\
\hline Asthenia & $19^{\mathrm{h}}$ & 50 & naive & no & ongoing $^{\mathrm{e}}$ & 1 & 300 & 2 \\
\hline Diarrhea & $19^{\mathrm{h}}$ & 50 & naive & no & NR & 1 & 300 & 2 \\
\hline Dysphagia & $20^{\mathrm{i}}$ & 60 & naive & yes & ongoing $^{\mathrm{e}}$ & 1 & 100 & 0.5 \\
\hline Eyelid ptosis & $21^{\mathrm{j}}$ & 71 & naive & no & 114 & 1 & 100 & 1 \\
\hline Headache & $19^{\mathrm{h}}$ & 50 & naive & no & NR & 1 & 300 & 2 \\
\hline $\begin{array}{c}\text { Lacrimation } \\
\text { increased }\end{array}$ & $19^{\mathrm{h}}$ & 50 & naive & no & NR & 1 & 300 & 2 \\
\hline
\end{tabular}


Table 4: (Continued)

\begin{tabular}{|c|c|c|c|c|c|c|c|c|}
\hline $\begin{array}{l}\text { Adverse event by } \\
\text { indication }^{\text {a }}\end{array}$ & Patient $^{\mathrm{a}}$ & Age & Treatment history & Serious event & $\begin{array}{c}\text { Symptom } \\
\text { duration (days) }\end{array}$ & No. injections $\mathbf{s}^{\mathrm{b}}$ & Dose $^{\mathrm{c}}$ (units) & Dilution ratio $^{\mathrm{d}}$ \\
\hline \multirow{3}{*}{$\begin{array}{c}\text { Muscular } \\
\text { weakness }\end{array}$} & $20^{k}$ & 72 & maint & no & 52 & 2 & 280 & 1 \\
\hline & $21^{\mathrm{i}}$ & 81 & maint & no & ongoing $^{\mathrm{e}}$ & 2 & $30-50$ & 1 \\
\hline & $22^{\mathrm{i}}$ & 62 & maint & no & 91 & 2 & 60 & 1 \\
\hline Nausea & $19^{\mathrm{h}}$ & 50 & naive & no & NR & 1 & 300 & 2 \\
\hline Pain & $19^{\mathrm{h}}$ & 50 & naive & no & ongoing $^{\mathrm{e}}$ & 1 & 300 & 2 \\
\hline Pyrexia & $20^{\mathrm{i}}$ & 60 & naive & no & ongoing $^{\mathrm{e}}$ & 3 & $40-150$ & 0.5 \\
\hline Tremor & $19^{\mathrm{h}}$ & 50 & naive & no & ongoing $^{\mathrm{e}}$ & 1 & 300 & 2 \\
\hline
\end{tabular}

$\mathrm{NA}=$ not applicable; $\mathrm{NR}=$ not reported.

${ }^{a}$ Patients counted once for each listed AE when multiple occurrences were reported; patient 2 (AFS) reported two muscular weakness AEs, and patient 6 (blepharospasm) reported two dysphonia adverse events; however, each was only counted once here. An arbitrary patient number is used to identify patients experiencing $\geq 1 \mathrm{AE}$.

${ }^{\mathrm{b}}$ Number of injections received prior to and including date of event onset.

${ }^{\mathrm{c}}$ Range of all doses administered prior to and including date of event onset.

${ }^{\mathrm{d}}$ Range of all dilutions used prior to and including date of event onset; calculated as volume of diluent added in mL per 100 units of onabotulinumtoxinA dose administered.

${ }^{\text {e}}$ Symptoms indicated as ongoing at the last treatment visit/end of study.

${ }^{\mathrm{f}}$ Patient treated for Meige's syndrome, including blepharospasm and spasmodic dysphonia (onabotulinumtoxinA into each thyroarytenoid muscle for spasmodic dysphonia outside of the MOBILITY study).

${ }^{\mathrm{g}}$ Resolved with sequelae.

${ }^{\text {h}}$ Patient treated with onabotulinumtoxinA for pain.

${ }^{\mathrm{i}}$ Patient treated with onabotulinumtoxinA for focal dystonia.

${ }^{\mathrm{j}}$ Patient treated with onabotulinumtoxinA for headache.

${ }^{\mathrm{k}}$ Patient treated with onabotulinumtoxinA for tremor.

across multiple therapeutic indications. MOBILITY is the longest study to date (approximately 5 years) to investigate the safety of onabotulinumtoxinA across multiple therapeutic indications by reporting the incidence rate of AEs in relation to the treatment.

Data were collected from patients who received between one and six treatments of onabotulinumtoxinA. Overall, onabotulinumtoxinA treatment was safe and well tolerated, with few discontinuations due to AEs $(n=6 / 704,0.9 \%)$ and no new safety signals.

\section{Very Long-Term Utilization and Safety}

Patients on maintenance onabotulinumtoxin A treatment had received as many as 90 treatments (mean $[\mathrm{SD}]=11$ [13]) for over 22 years prior to entering the study and showed no notable differences in the number of TRAEs reported when compared to the naive population, indicating no increased safety risk with chronic use. Earlier reports of long-term safety have agreed with this finding and have even indicated that the incidence of AEs at times decreases with increased treatment duration. ${ }^{11,12}$ This, of course, is an important observation as these indications are chronic conditions requiring repeat treatments with onabotulinumtoxinA over the long term.

\section{Dosing and Safety}

The mean dose administered was $221 \mathrm{U}$ (median, $200 \mathrm{U}$ ) of onabotulinumtoxinA, which is reflective of real-world clinical practice (Figure 2). A total of 280 patients were treated with
$>360 \mathrm{U}$ of onabotulinumtoxinA during the study, and there did not appear to be any correlation between high doses and an increased incidence of TRAEs; indeed, there was no statistically significant association between dose of onabotulinumtoxinA at baseline and the incidence of TRAEs. Overall, from the safety population of 1372 patients, the majority of TRAEs were reported by patients who had received $<360 \mathrm{U}$ (within a 3-month period) with only two patients reporting TRAEs following administration of high doses of onabotulinumtoxinA (400 U and $500 \mathrm{U}$ ).

In addition to examining the correlation between dose and incidence of AEs, the relationship of age and AEs was explored, as onabotulinumtoxinA is indicated for a variety of diseases spanning a wide range of age groups. The data show an overall trend of increasing incidence of AEs with increasing age. This finding is not unexpected as older patients often suffer from additional comorbidities and associated AEs. ${ }^{13}$

The most commonly reported TRAE in this study was muscular weakness $(n=8 / 1372,0.6 \%)$ which is consistent with previously published findings, the adverse reactions outlined in the product label, and the known mechanism of action of onaboutlinumtoxinA. ${ }^{5,6,11,12,14-17}$ The reports of muscular weakness are to be expected as onabotulinumtoxinA acts by locally reducing acetylcholine release at neuromuscular junctions. A single incidence was reported as a TRAE considered related to the injection procedure rather than the therapeutic treatment itself. The reported TRAE was herpes zoster in one AFS patient, where symptoms were likely exacerbated by the injection procedure. ${ }^{18}$ 
In addition to the impact of the disease condition on AEs, there is significant heterogeneity in patients with neurological disorders that may also influence incidence of AEs.

Limited safety data are available from long-term, prospective, observational studies with onabotulinumtoxinA. Overall, results from MOBILITY are comparable to existing published reports from studies with similar designs and with the product label. ${ }^{, 19,20}$

\section{LiMitaTiONS}

Although our study was able to capture long-term safety information across a number of indications in real-world clinical practice, several limitations should be noted. Overall, a large proportion of patients in the safety cohort discontinued the study $(n=704 / 1372,51 \%)$ for various reasons including "other" (363/704, 52\%), comprising mostly study site closure, investigator study discontinuations, or limited availability of resources (i.e., clinic nurse) at the participating clinic; lost to follow-up (195/704, 28\%); and patient withdrawal of consent (92/704, $13 \%)$. Study discontinuations grouped under "other" were primarily due to study site closure, possibly limiting the generalizability of the findings as data are biased toward those patients who remained in the study. Investigators were required to monitor and report all AEs based on their clinical assessment; however, there was no formalized direct questioning for AEs nor was there any required laboratory testing as may be more typical in a controlled clinical trial. This may mean that all details regarding an AE may not have been captured, including data pertaining to disease severity and comorbidities, which may have helped to further characterize and understand the reported AEs. In addition, reports on concomitant medications were only captured for the condition in which they were being treated with onabotulinumtoxinA; therefore, not all concomitant medication information was captured. Furthermore, information regarding physician experience with administering onabotulinumtoxinA may have been helpful in determining whether there was any correlation to the reported AEs. Similarly, information regarding the specific muscles injected would have been helpful to classify AEs as local or due to the distal spread of the toxin.

Beyond local effects, the distant spread of the toxin is a concern when evaluating the safety of onabotulinumtoxinA treatment. Reports have suggested that botulinum toxin treatment has the potential to spread beyond the local injection site, causing symptoms consistent with botulism. ${ }^{21-23}$ These concerns have been raised by the EMA, FDA, and Health Canada, prompting the inclusion of a warning in product labeling to acknowledge the risk of the effect of the distal spread of toxin. ${ }^{24-26}$ Although the concern is recognized, data regarding the specific muscles injected were not collected in this study, and therefore, we were not able to report whether AEs were possibly due to local or distant spread of the toxin.

\section{Conclusion}

In patients with follow-up for up to six treatments, TRAEs were reported in $<2 \%$ of the safety population over the course of nearly 5 years. Overall, our findings suggest that treatment with onabotulinumtoxinA is safe for long-term use across a variety of therapeutic indications.

\section{STATEMENT of Authorship}

All authors met the ICMJE authorship criteria. Neither honoraria nor payments were made for authorship. Conceptualized and designed the study: MB, TW. Designed the data collection instruments: MB. Carried out the initial analysis: MB, TW. Coordinated and supervised data collection: MB, GT, RB, TW. Reviewed and revised the manuscript: TW, MJ, MB, SD, RM, FI, RB, GT. Approved the final manuscript as submitted: TW, MJ, MB, SD, RM, FI, RB, GT.

\section{CONFLiCTS OF INTEREST}

TW has received research funds from Allergan plc, has been a member of an advisory board, steering committee, and mentoring program for Allergan plc, and has been a member of advisory boards for Ipsen and Merz. MJ has participated in speaking engagements and advisory boards with Allergan, Merz, Novartis, Abbvie, and Teva and has received research grants from CIHR, AMOSO, MITACS, and NCE. MB is an employee of Allergan. SD was formerly a full-time employee of Allergan plc during the conduct, analysis, and publication of this study. RM has been a member of a scientific advisory committee for Allergan and has been a speaker/consultant for Merz. FI has received honoraria and research funds from Allergan plc and Merz Pharma Canada. RB has no conflicts of interest to declare. GT was a former employee of Allergan plc.

\section{ACKNOWLEDGEMENTS}

This study was sponsored by Allergan, Inc. The authors thank Merlin Njoya of McKesson for statistical support; Amy Kuang, $\mathrm{PhD}$, of Allergan plc for writing and editorial support for this manuscript; and Aleksej Zuzek, PhD, of Allergan plc (Irvine, CA) for his critical review of an earlier draft of this manuscript.

\section{REFERENCES}

1. Aoki KR. Botulinum toxin: a successful therapeutic protein. Curr Med Chem. 2004;11(23):3085-92.

2. Schantz EJ, Johnson EA. Properties and use of botulinum toxin and other microbial neurotoxins in medicine. Microbiol Rev. 1992;56(1):80-99.

3. Jankovic J. Botulinum toxin: state of the art. Mov Disord. 2017;32(8):1131-8.

4. Aoki KR, Francis J. Updates on the antinociceptive mechanism hypothesis of botulinum toxin A. Parkinsonism Relat Disord. 2011;17(suppl 1):S28-33.

5. Burstein R, Zhang X, Levy D, Aoki KR, Brin MF. Selective inhibition of meningeal nociceptors by botulinum neurotoxin type A: therapeutic implications for migraine and other pains. Cephalalgia. 2014;34(11):853-69.

6. Allergan. PrBOTOX ${ }^{\circledR}$ onabotulinumtoxinA for injection $\mathrm{Ph}$. Eur. Clostridium botulinum type A neurotoxin complex (900kD) Sterile vacuum-dried concentrate powder for solution for injection 50, 100 and 200 Allergan units per vial. Markham, ON L6G 0B5: Allergan, Inc; 2016.

7. BOTOX ${ }^{\circledR}$ onabotulinumtoxinA for injection. Product monograph. Summary of product characteristics. Irvine, CA, USA: Allergan plc; 2016.

8. PrBOTOX $^{\circledR}$ (onabotulinumtoxinA for injection Ph. Eur. Clostridium botulinum type A neurotoxin complex). Full prescribing information. Markham, ON, Canada: Allergan, Inc; 2016.

9. Nuanthaisong U, Abraham N, Goldman HB. Incidence of adverse events after high doses of onabotulinumtoxinA for multiple indications. Urology. 2014;84(5):1044-8. 
10. Introductory Guide, MedDRA Version 15.1. Geneva, Switzerland: International Conference on Harmonisation; 2012. MSSO-DI6003-15.1.0.

11. Albavera-Hernandez C, Rodriguez JM, Idrovo AJ. Safety of botulinum toxin type A among children with spasticity secondary to cerebral palsy: a systematic review of randomized clinical trials. Clin Rehabil. 2009;23(5):394-407.

12. Brashear A. The safety and tolerability of botulinum toxins for the treatment of cervical dystonia. Expert Opin Drug Saf. 2005; 4(2):241-9.

13. Wolff JL, Starfield B, Anderson G. Prevalence, expenditures, and complications of multiple chronic conditions in the elderly. Arch Intern Med. 2002;162(20):2269-76.

14. Defazio G, Abbruzzese G, Girlanda P, et al. Botulinum toxin A treatment for primary hemifacial spasm: a 10-year multicenter study. Arch Neurol. 2002;59(3):418-20.

15. Lagalla G, Danni M, Reiter F, Ceravolo MG, Provinciali L. Poststroke spasticity management with repeated botulinum toxin injections in the upper limb. Am J Phys Med Rehabil. 2000; 79(4):377-84.

16. Mejia NI, Vuong KD, Jankovic J. Long-term botulinum toxin efficacy, safety, and immunogenicity. Mov Disord. 2005; 20(5):592-7.

17. Russo RN, Crotty M, Miller MD, Murchland S, Flett P, Haan E. Upper-limb botulinum toxin A injection and occupational therapy in children with hemiplegic cerebral palsy identified from a population register: a single-blind, randomized, controlled trial. Pediatrics. 2007;119(5):e1149-58.

18. Gadient PM, Smith JH, Ryan SJ. Herpes zoster ophthalmicus following onabotulinumtoxinA administration for chronic migraine: a case report and literature review. Cephalalgia. 2015; 35(5):443-8.
19. Misra VP, Ehler E, Zakine B, Maisonobe P, Simonetta-Moreau M, Interest in $\mathrm{CD}$ group. Factors influencing response to Botulinum toxin type $\mathrm{A}$ in patients with idiopathic cervical dystonia: results from an international observational study. BMJ Open. 2012; 2(e000881).

20. Muller F, Cugy E, Ducerf C, et al. Safety and self-reported efficacy of botulinum toxin for adult spasticity in current clinical practice: a prospective observational study. Clin Rehabil. 2012;26(2): 174-9.

21. Bakheit AM, Ward CD, McLellan DL. Generalised botulism-like syndrome after intramuscular injections of botulinum toxin type A: a report of two cases. J Neurol Neurosurg Psychiatry. 1997; 62(2):198

22. Cobb DB, Watson WA, Fernandez MC. Botulism-like syndrome after injections of botulinum toxin. Vet Hum Toxicol. 2000; 42(3): 163

23. Tugnoli V, Eleopra R, Quatrale R, Capone JG, Sensi M, Gastaldo E. Botulism-like syndrome after botulinum toxin type A injections for focal hyperhidrosis. $\mathrm{Br} \mathrm{J}$ Dermatol. 2002;147(4):808-9.

24. Botox ${ }^{\circledR}$ (onabotulinumtoxinA for injection). Summary of product characteristics. Westport, County Mayo, Ireland: Allergan Pharmaceuticals Ireland; 2014

25. Health Canada. Botulinum toxin type A (Botox) and distant toxin spread. Canadian Adverse Reaction Newsletter. 2008;2016(4): $1-2$.

26. US Food and Drug Administration. OnabotulinumtoxinA (marketed as Botox/Botox Cosmetic), abobotulinumtoxinA (marketed as Dysport) and rimabotulinumtoxinB (marketed as Myobloc) information. Available at: http://www.fda.gov/Drugs/DrugSafety/ PostmarketDrugSafetyInformationforPatientsandProviders/ucm 175011.htm. accessed December 13, 2017. 